\title{
The natural liftings of connections to tensor powers of the cotangent bundle
}

\author{
Jan Kurek and Wlodzimierz M. Mikulski
}




\title{
THE NATURAL LIFTINGS OF CONNECTIONS TO TENSOR POWERS OF THE COTANGENT BUNDLE
}

\author{
JAN KUREK AND WŁODZIMIERZ M. MIKULSKI
}

\begin{abstract}
We reduce the problem of finding all $\mathcal{M} f_{m}$-natural operators $B: Q \rightsquigarrow Q\left(\otimes^{k} T^{*}\right)$ lifting classical linear connections $\nabla$ on $m$-manifolds $M$ into classical linear connections $B_{M}(\nabla)$ on the $k$ th tensor power $\otimes^{k} T^{*} M$ of the cotangent bundle $T^{*} M$ of $M$ to the fairly known description of all $\mathcal{M} f_{m}$-natural operators $D: Q \rightsquigarrow \otimes^{p} T^{*} \otimes \otimes^{q} T$ transforming classical linear connections $\nabla$ on $m$-manifolds $M$ into tensor fields $D_{M}(\nabla)$ of types $(q, p)$ on $M$.
\end{abstract}

\section{INTRODUCTION}

All manifolds and maps are assumed to be smooth (of class $C^{\infty}$ ). All manifolds are assumed to be Hausdorff, finite dimensional and without boundaries. The category of $m$-dimensional manifolds and their embeddings is denoted by $\mathcal{M} f_{m}$.

In [3], M. Kureš described completely all $\mathcal{M} f_{m}$-natural operators $B: Q \rightsquigarrow Q T^{*}$ lifting classical linear connections $\nabla$ on $m$-manifolds $M$ into classical linear connections $B_{M}(\nabla)$ on the cotangent bundle $T^{*} M$ of $M$.

In the present note, we study a similar problem for $\mathcal{M} f_{m}$-natural operators $B$ : $Q \rightsquigarrow Q\left(\otimes^{k} T^{*}\right)$ transforming classical linear connections $\nabla$ on $m$-manifolds $M$ into classical linear connections $B_{M}(\nabla)$ on the $k$ th tensor power $\otimes^{k} T^{*} M$ of $T^{*} M$.

An example of an $\mathcal{M} f_{m}$-natural operator $Q \rightsquigarrow Q\left(\otimes^{k} T^{*}\right)$ is the following construction. Let $\nabla$ be a classical linear connection on $M$. We extend $\nabla$ to the linear connection $D=\otimes^{k} \nabla^{*}$ in the vector bundle $E=\otimes^{k} T^{*} M \rightarrow M$. Then, owing to [1], we have a classical linear connection (we will denote it by $\nabla^{(k)}$ ) on $E=\otimes^{k} T^{*} M$ corresponding to $(D, \nabla)$.

The main result of the paper says that if $k \geq 3$, then the $\mathcal{M} f_{m}$-natural operators $B: Q \rightsquigarrow Q\left(\otimes^{k} T^{*}\right)$ are in bijection with the systems $D=\left(D_{j}\right)_{j=1, \ldots, 5}$ of $\mathcal{M} f_{m}$-natural operators $D_{j}: Q \rightsquigarrow \otimes^{p_{j}} T^{*} \otimes \otimes^{q_{j}} T$ transforming classical linear connections $\nabla$ on $m$-manifolds $M$ into tensor fields of types $\left(q_{1}, p_{1}\right)=(1,2)$, $\left(q_{2}, p_{2}\right)=(0, k+2),\left(q_{3}, p_{3}\right)=(k, k+2)$ and $\left(q_{4}, p_{4}\right)=\left(q_{5}, p_{5}\right)=(k, k+1)$ on $M$. The case of $k=2$ is also studied.

Such a characterization is sufficient because a complete description of all $\mathcal{M} f_{m^{-}}$ natural operators $Q \rightsquigarrow \otimes^{p} T^{*} \otimes \otimes^{q} T$ is fairly known (see Remark 1). 
A general concept of natural operators can be found in [2]. In this paper, we will use some partial definitions of natural operators only. In Section 2, we recall these partial definitions for the readers' convenience. In Sections 3 and 4, we made some preparations. The main theorem is presented in Section 5.

\section{BASIC DEFINITIONS}

We will use the following partial definitions of natural operators only. (The general concept of natural operators can be found in [2].)

Definition 1. An $\mathcal{M} f_{m}$-natural operator $B: Q \rightsquigarrow Q\left(\otimes^{k} T^{*}\right)$ transforming classical linear connections $\nabla$ on $m$-manifolds $M$ into classical linear connections $B_{M}(\nabla)$ on $\otimes^{k} T^{*} M$ is an $\mathcal{M} f_{m}$-invariant system $B=\left\{B_{M}\right\}_{M \in \operatorname{obj}\left(\mathcal{M} f_{m}\right)}$ of regular operators (functions)

$$
B_{M}: \underline{Q}(M) \rightarrow \underline{Q}\left(\otimes^{k} T^{*} M\right)
$$

for any $m$-manifold $M$, where $Q(M)$ is the set of all classical linear connections on $M$. The $\mathcal{M} f_{m}$-invariance of $B$ means that if $\nabla_{1} \in \underline{Q}\left(M_{1}\right)$ and $\nabla_{2} \in \underline{Q}\left(M_{2}\right)$ are $\varphi$-related by an embedding $\varphi: M_{1} \rightarrow M_{2}$ between $m$-manifolds (i. e., $\varphi$ is $\left(\nabla_{1}, \nabla_{2}\right)$ affine), then $B_{M_{1}}\left(\nabla_{1}\right)$ and $B_{M_{2}}\left(\nabla_{2}\right)$ are $\otimes^{k} T^{*} \varphi$-related. The regularity of $B$ means that $B_{M}$ transforms smoothly parametrized families of connections into smoothly parametrized ones.

For example, the family $B^{(k)}: Q \rightsquigarrow Q\left(\otimes^{k} T^{*}\right)$ of operators

$$
B_{M}^{(k)}: \underline{Q}(M) \rightarrow \underline{Q}\left(\otimes^{k} T^{*} M\right), \quad B_{M}^{(k)}(\nabla):=\nabla^{(k)}, \quad \nabla \in \underline{Q}(M)
$$

(see Section 1), for any $m$-manifolds $M$, is an $\mathcal{M} f_{m}$-natural operator.

Definition 2. An $\mathcal{M} f_{m}$-natural operator $C: Q \rightsquigarrow\left(\otimes^{k} T^{*}, \otimes^{p} T^{*} \otimes \otimes^{q} T\right)$ transforming classical linear connections $\nabla$ on $m$-manifolds $M$ into base preserving fibred maps $C_{M}(\nabla): \otimes^{k} T^{*} M \rightarrow \otimes^{p} T^{*} M \otimes \otimes^{q} T M$ is an $\mathcal{M} f_{m}$-invariant system $C=\left\{C_{M}\right\}_{M \in \operatorname{obj}\left(\mathcal{M} f_{m}\right)}$ of regular operators

$$
C_{M}: \underline{Q}(M) \rightarrow C_{M}^{\infty}\left(\otimes^{k} T^{*} M, \otimes^{p} T^{*} M \otimes \otimes^{q} T M\right)
$$

for any $m$-manifold $M$, where $\underline{Q}(M)$ is as in Definition 1 and

$$
C_{M}^{\infty}\left(\otimes^{k} T^{*} M, \otimes^{p} T^{*} M \otimes \otimes^{q} T M\right)
$$

is the set of all base preserving fibred maps $\otimes^{k} T^{*} M \rightarrow \otimes^{p} T^{*} M \otimes \otimes^{q} T M$. The $\mathcal{M} f_{m}$-invariance of $C$ means that if $\nabla_{1} \in \underline{Q}\left(M_{1}\right)$ and $\nabla_{2} \in \underline{Q}\left(M_{2}\right)$ are $\varphi$ related by an embedding $\varphi: M_{1} \rightarrow M_{2}$ between $m$-manifolds, then fibred maps $C_{M_{1}}\left(\nabla_{1}\right): \otimes^{k} T^{*} M_{1} \rightarrow \otimes^{p} T^{*} M_{1} \otimes \otimes^{q} T M_{1}$ and $C_{M_{2}}\left(\nabla_{2}\right): \otimes^{k} T^{*} M_{2} \rightarrow$ $\otimes^{p} T^{*} M_{2} \otimes \otimes^{q} T M_{2}$ are also $\varphi$-related (i.e., $C_{M_{2}}\left(\nabla_{2}\right) \circ \otimes^{k} T^{*} \varphi=\left(\otimes^{p} T^{*} \varphi \otimes\right.$ $\left.\left.\otimes^{q} T \varphi\right) \circ C_{M_{1}}\left(\nabla_{1}\right)\right)$. The regularity means almost the same as in Definition 1. 
For example, the curvature tensor $\mathcal{R}(\nabla)$ of a classical linear connection $\nabla$ determines an $\mathcal{M} f_{m}$-natural operator $C^{\mathcal{R}}: Q \rightsquigarrow\left(\otimes^{k} T^{*}, \otimes^{3} T^{*} \otimes T\right)$. More precisely, the family $C^{\mathcal{R}}: Q \rightsquigarrow\left(\otimes^{k} T^{*}, \otimes^{3} T^{*} \otimes T M\right)$ of operators $C_{M}^{\mathcal{R}}: \underline{Q}(M) \rightarrow$ $C_{M}^{\infty}\left(\otimes^{k} T^{*} M, \otimes^{3} T^{*} M \otimes T M\right)$ given by

$$
C_{M}^{\mathcal{R}}(\nabla):=\mathcal{R}(\nabla) \circ \pi_{M}: \otimes^{k} T^{*} M \rightarrow \otimes^{3} T^{*} M \otimes T M, \nabla \in \underline{Q}(M)
$$

(where $\pi_{M}: \otimes^{k} T^{*} M \rightarrow M$ is the bundle projection), for any $m$-manifold $M$, is an $\mathcal{M} f_{m}$-natural operator.

Definition 3. An $\mathcal{M} f_{m}$-natural transformation $C: \otimes^{k} T^{*} \rightarrow \otimes^{p} T^{*} \otimes \otimes^{q} T$ is an $\mathcal{M} f_{m}$-natural operator $C=\left\{C_{M}\right\}: Q \rightsquigarrow\left(\otimes^{k} T^{*}, \otimes^{p} T^{*} \otimes \otimes^{q} T\right)$ such that $C_{M}: Q(M) \rightarrow C_{M}^{\infty}\left(\otimes^{k} T^{*} M, \otimes^{p} T^{*} M \otimes \otimes^{q} T M\right)$ is a constant function for any $M \in \operatorname{obj}\left(\mathcal{M} f_{m}\right)$.

For example, the family $C: \otimes^{k} T^{*} \rightarrow \otimes^{2 k} T^{*}$ of fibred maps $C_{M}: \otimes^{k} T^{*} M \rightarrow$ $\otimes{ }^{2 k} T^{*} M$ given by

$$
C_{M}(v):=v \otimes v, v \in \otimes^{k} T_{x}^{*} M, x \in M,
$$

for any $m$-manifold $M$, is an $\mathcal{M} f_{m}$-natural transformation.

Definition 4. An $\mathcal{M} f_{m}$-natural operator ( $\mathcal{M} f_{m}$-natural tensor) $D: Q \rightsquigarrow \otimes^{p} T^{*} \otimes$ $\otimes^{q} T$ transforming classical linear connections $\nabla$ on $m$-manifolds $M$ into tensor fields $D_{M}(\nabla)$ of type $\otimes^{p} T^{*} \otimes \otimes^{q} T$ on $M$ is an $\mathcal{M} f_{m}$-invariant system of regular operators

$$
D_{M}: \underline{Q}(M) \rightarrow C_{M}^{\infty}\left(\otimes^{p} T^{*} M \otimes \otimes^{q} T M\right)
$$

for any $m$-manifold $M$, where $C_{M}^{\infty}\left(\otimes^{p} T^{*} M \otimes \otimes^{q} T M\right)$ is the space of all tensor fields of type $\otimes^{p} T^{*} \otimes \otimes^{q} T$ on $M$. The $\mathcal{M} f_{m}$-invariance of $D$ means that if $\nabla_{1} \in$ $Q\left(M_{1}\right)$ and $\nabla_{2} \in Q\left(M_{2}\right)$ are $\varphi$-related by an embedding $\varphi: M_{1} \rightarrow M_{2}$, then $\bar{D}_{M_{1}}\left(\nabla_{1}\right)$ and $D_{M_{2}}\left(\nabla_{2}\right)$ are also $\varphi$-related (i.e., $\left(\otimes^{p} T^{*} \varphi \otimes \otimes^{q} T \varphi\right) \circ D_{M_{1}}\left(\nabla_{1}\right)=$ $\left.D_{M_{2}}\left(\nabla_{2}\right) \circ \varphi\right)$. The regularity means almost the same as in Definition 1.

For example, the family $\mathcal{R}: Q \rightsquigarrow \otimes^{3} T^{*} \otimes T$ of operators $\mathcal{R}_{M}: \underline{Q}(M) \rightarrow$ $C_{M}^{\infty}\left(\otimes^{3} T^{*} M \otimes \otimes T M\right)$ given by

$$
\mathcal{R}_{M}(\nabla)=\text { the curvature tensor of } \nabla \in \underline{Q}(M),
$$

for any $m$-manifold $M$, is an $\mathcal{M} f_{m}$-natural operator.

Remark 1. A full description of all $\mathcal{M} f_{m}$-natural operators $Q_{\tau} \rightsquigarrow \otimes^{p} T^{*} \otimes \otimes^{q} T$ transforming torsion free classical connections on $m$-manifolds into tensor fields of types $(q, p)$ can be found in the Lemma in in [2, Section 33.4]. The following is the description. Each covariant derivative of the curvature

$$
\mathcal{R}(\nabla) \in C_{M}^{\infty}\left(\wedge^{2} T^{*} M \otimes T^{*} M \otimes T M\right)
$$


of a classical linear connection $\nabla$ is an $\mathcal{M} f_{m}$-natural tensor. Further, every tensor multiplication of two natural tensors and every contraction on one covariant and one contravariant entry of a natural tensor give a new natural tensor. Finally, we can tensor any natural tensor with a connection independent natural tensor, we can permute any number of entries in the tensor product and we can repeat these steps and take linear combinations. In this way, we can obtain any natural tensor of types $(q, p)$ depending on a torsion free classical linear connection. Modifying the proof of the above fact, one can see that all natural tensors of a (not necessarily torsion free) classical linear connection $\nabla$ can be obtained, provided we also include the torsion tensor $\mathcal{T}(\nabla)$ and their covariant derivatives to the above procedure.

\section{A PROPOSITION}

We have the following description of $\mathcal{M} f_{m}$-natural operators $Q \rightsquigarrow Q\left(\otimes^{k} T^{*}\right)$ by means of systems of $\mathcal{M} f_{m}$-natural operators $Q \rightsquigarrow\left(\otimes^{k} T^{*}, \otimes^{p} T^{*} \otimes \otimes^{q} T\right)$.

Proposition 1. The $\mathcal{M} f_{m}$-natural operators $B: Q \rightsquigarrow Q\left(\otimes^{k} T^{*}\right)$ are in bijection with the systems $C=\left(C_{j}\right)_{j=1, \ldots, 8}$ of $\mathcal{M} f_{m}$-natural operators $C_{1}: Q \rightsquigarrow$ $\left(\otimes^{k} T^{*}, \otimes^{2} T^{*} \otimes T\right), C_{2}: Q \rightsquigarrow\left(\otimes^{k} T^{*}, T^{*} \otimes \otimes^{k+1} T\right), C_{3}: Q \rightsquigarrow\left(\otimes^{k} T^{*}, T^{*} \otimes\right.$ $\left.\otimes^{k+1} T\right), C_{4}: Q \rightsquigarrow\left(\otimes^{k} T^{*}, \otimes^{2 k+1} T\right), C_{5}: Q \rightsquigarrow\left(\otimes^{k} T^{*}, \otimes^{k+2} T^{*}\right), C_{6}:$ $Q \rightsquigarrow\left(\otimes^{k} T^{*}, \otimes^{k+1} T^{*} \otimes \otimes^{k} T\right), C_{7}: Q \rightsquigarrow\left(\otimes^{k} T^{*}, \otimes^{k+1} T^{*} \otimes \otimes^{k} T\right)$ and $C_{8}: Q \rightsquigarrow\left(\otimes^{k} T^{*}, \otimes^{k} T^{*} \otimes \otimes^{2 k} T\right)$.

Proof. Let $\nabla$ be a classical linear connection on an $m$-manifold $M$. Then, for any point $v \in \otimes^{k} T_{x}^{*} M, x \in M$, we have the standard linear isomorphisms

$$
T_{v}\left(\otimes^{k} T^{*} M\right) \tilde{=} H_{v}^{\nabla} \oplus V_{v}\left(\otimes^{k} T^{*} M\right) \tilde{=} T_{x} M \oplus\left(\otimes^{k} T_{x}^{*} M\right)
$$

canonically depending on $\nabla$, where $H_{v}^{\nabla} \subset T_{v}\left(\otimes^{k} T^{*} M\right)$ is the $\nabla$-horizontal subspace and $V_{v}\left(\otimes^{k} T^{*} M\right) \subset T_{v}\left(\otimes^{k} T^{*} M\right)$ is the vertical subspace. So, by an obvious linear algebra, we have the identification (linear isomorphism)

$$
\begin{aligned}
T_{v}^{*} & \left(\otimes^{k} T^{*} M\right) \otimes T_{v}^{*}\left(\otimes^{k} T^{*} M\right) \otimes T_{v}\left(\otimes^{k} T^{*} M\right) \tilde{=} \\
& \left(T_{x}^{*} M \otimes T_{x}^{*} M \otimes T_{x} M\right) \oplus\left(T_{x}^{*} M \otimes\left(\otimes^{k} T_{x}^{*} M\right)^{*} \otimes T_{x} M\right) \oplus \\
& \left(\left(\otimes^{k} T_{x}^{*} M\right)^{*} \otimes T_{x}^{*} M \otimes T_{x} M\right) \oplus\left(\left(\otimes^{k} T_{x}^{*} M\right)^{*} \otimes\left(\otimes^{k} T_{x}^{*} M\right)^{*} \otimes T_{x} M\right) \oplus \\
& \left(T_{x}^{*} M \otimes T_{x}^{*} M \otimes \otimes^{k} T_{x}^{*} M\right) \oplus\left(T_{x}^{*} M \otimes\left(\otimes^{k} T_{x}^{*} M\right)^{*} \otimes \otimes^{k} T_{x}^{*} M\right) \oplus \\
& \left(\left(\otimes^{k} T_{x}^{*} M\right)^{*} \otimes T_{x}^{*} M \otimes \otimes^{k} T_{x}^{*} M\right) \oplus\left(\left(\otimes^{k} T_{x}^{*} M\right)^{*} \otimes\left(\otimes^{k} T_{x}^{*} M\right)^{*} \otimes \otimes^{k} T_{x}^{*} M\right)
\end{aligned}
$$


canonically depending on $\nabla$ or (simply) the one

$$
\begin{aligned}
T_{v}^{*}\left(\otimes^{k} T^{*} M\right) \otimes & T_{v}^{*}\left(\otimes^{k} T^{*} M\right) \otimes T_{v}\left(\otimes^{k} T^{*} M\right) \tilde{=} \\
& \left(\otimes^{2} T_{x}^{*} M \otimes T_{x} M\right) \oplus\left(T_{x}^{*} M \otimes \otimes^{k+1} T_{x} M\right) \oplus \\
& \left(T_{x}^{*} M \otimes \otimes^{k+1} T_{x} M\right) \oplus\left(\otimes^{2 k+1} T_{x} M\right) \oplus \\
& \left(\otimes^{k+2} T_{x}^{*} M\right) \oplus\left(\otimes^{k+1} T_{x}^{*} M \otimes \otimes^{k} T_{x} M\right) \oplus \\
& \left(\otimes^{k+1} T_{x}^{*} M \otimes \otimes^{k} T_{x} M\right) \oplus\left(\otimes^{k} T_{x}^{*} M \otimes \otimes^{2 k} T_{x} M\right) .
\end{aligned}
$$

Consequently, any $\mathcal{M} f_{m}$-natural operator $B: Q \rightsquigarrow Q\left(\otimes^{k} T^{*} M\right)$ can be identified with the system $C=\left(C_{i}\right)_{i=1, \ldots, 8}$ of $\mathcal{M} f_{m}$-natural operators $C_{1}: Q \rightsquigarrow$ $\left(\otimes^{k} T^{*}, \otimes^{2} T^{*} \otimes T\right), C_{2}: Q \rightsquigarrow\left(\otimes^{k} T^{*}, T^{*} \otimes \otimes^{k+1} T\right), C_{3}: Q \rightsquigarrow\left(\otimes^{k} T^{*}, T^{*} \otimes\right.$ $\left.\otimes^{k+1} T\right), C_{4}: Q \rightsquigarrow\left(\otimes^{k} T^{*}, \otimes^{2 k+1} T\right), C_{5}: Q \rightsquigarrow\left(\otimes^{k} T^{*}, \otimes^{k+2} T^{*}\right), C_{6}:$ $Q \rightsquigarrow\left(\otimes^{k} T^{*}, \otimes^{k+1} T^{*} \otimes \otimes^{k} T\right), C_{7}: Q \rightsquigarrow\left(\otimes^{k} T^{*}, \otimes^{k+1} T^{*} \otimes \otimes^{k} T\right)$ and $C_{8}: Q \rightsquigarrow\left(\otimes^{k} T^{*}, \otimes^{k} T^{*} \otimes \otimes^{2 k} T\right)$ defined by

$$
\left(\left(C_{i}\right)_{M}(\nabla)(v)\right)_{i=1, \ldots ., 8}=\delta_{M}(\nabla)(v)
$$

modulo the last identification, $v \in \otimes^{k} T_{x}^{*} M, x \in M$, where $\delta_{M}(\nabla): \otimes^{k} T^{*} M \rightarrow$ $T^{*}\left(\otimes^{k} T^{*} M\right) \otimes T^{*}\left(\otimes^{k} T^{*} M\right) \otimes T\left(\otimes^{k} T^{*} M\right)$ is the tensor field of type $T^{*} \otimes T^{*} \otimes T$ on $\otimes^{k} T^{*} M$ with $B_{M}(\nabla)=\nabla^{(k)}+\delta_{M}(\nabla)$. Here, $\nabla^{(k)}$ are the classical linear connections on $\otimes^{k} T^{*} M$ corresponding to $\left(\otimes^{k} \nabla^{*}, \nabla\right)$ as in Section 1.

The proof of the proposition is complete.

\section{SOME LEMMAS}

To obtain a description of $\mathcal{M} f_{m}$-natural operators $Q \rightsquigarrow Q\left(\otimes^{k} T^{*}\right)$ by means of systems of $\mathcal{M} f_{m}$-natural operators $Q \rightsquigarrow \otimes^{p} T^{*} \otimes \otimes^{q} T$, we prove some lemmas.

Lemma 1. If $q>p$, then any $\mathcal{M} f_{m}$-natural operator $C: Q \rightsquigarrow\left(\otimes^{k} T^{*}, \otimes^{p} T^{*} \otimes\right.$ $\left.\otimes^{q} T\right)$ is the zero one (i.e., $C_{M}(\nabla)(v)=0$ for all $M \in \operatorname{obj}\left(\mathcal{M} f_{m}\right), \nabla \in Q(M)$, $\left.v \in \otimes^{k} T_{x}^{*} M, x \in M\right)$.

Proof. We have to show that $C_{M}(\nabla)(v)=0 \in \otimes^{p} T_{x}^{*} M \otimes \otimes^{q} T_{x} M$ for all $m$ manifolds $M, \nabla \in \underline{Q}(M)$ and $v \in \otimes^{k} T_{x}^{*} M, x \in M$. Using the invariance of $C$ with respect to the manifold charts, we may assume $M=\mathbb{R}^{m}, x=0$.

By the non-linear Petree theorem (see [2]), one can assume that the Christoffel symbols $\nabla_{j k}^{i}$ of $\nabla$ in the usual coordinates on $\mathbb{R}^{m}$ are polynomials (of arbitrary degree $d$ ) with coefficients $\nabla_{j k ; \alpha}^{i}$, i. e.

$$
\nabla=\left(\nabla_{j k ; \alpha}^{i}\right)_{i, j, k=1, \ldots, m, \alpha \in \mathbf{N}^{m},|\alpha| \leq d}
$$

or in short $\nabla=\left(\nabla_{j k ; \alpha}^{i}\right)$. The regularity of $C$ yields that $C_{\mathbb{R}^{m}}\left(\left(\nabla_{j k ; \alpha}^{i}\right)\right)(v)$ is smooth in $\left(\left(\nabla_{j k ; \alpha}^{i}\right), v\right)$ for any (fixed) $d$. 
Using the invariance of $C$ with respect to the homotheties $\frac{1}{t} i d_{\mathbb{R}^{m}}$ for $t>0$, we get the homogeneity condition

$$
C_{\mathbb{R}^{m}}\left(\left(\nabla_{j k ; \alpha}^{i}\right)\right)(v)=t^{q-p} C_{\mathbb{R}^{m}}\left(\left(t^{1+|\alpha|} \nabla_{j k ; \alpha}^{i}\right)\right)\left(t^{k} v\right) .
$$

Putting $t \rightarrow 0$, we get $C_{\mathbb{R}^{m}}\left(\left(\nabla_{j k ; \alpha}^{i}\right)\right)(v)=0$ as $q-p>0$.

Lemma 2. If $k \geq 2$, the $\mathcal{M} f_{m}$-natural operators $C: Q \rightsquigarrow\left(\otimes^{k} T^{*}, \otimes^{q+1} T^{*} \otimes\right.$ $\left.\otimes^{q} T\right)$ are in bijection with the $\mathcal{M} f_{m}$-natural operators $D: Q \rightsquigarrow \otimes^{q+1} T^{*} \otimes \otimes^{q} T$.

More precisely, if $k \geq 2$, any $\mathcal{M} f_{m}$-natural operator $C: Q \rightsquigarrow\left(\otimes^{k} T^{*}, \otimes^{q+1} T^{*}\right.$ $\left.\otimes \otimes^{q} T\right)$ defines an $\mathcal{M} f_{m}$-natural operator $D^{C}: Q \rightsquigarrow \otimes^{q+1} T^{*} \otimes \otimes^{q} T$,

$$
D_{M}^{C}(\nabla)(x):=C_{M}(\nabla)(0),
$$

$0 \in \otimes^{k} T_{x}^{*} M$ (the zero element), $x \in M, M \in \operatorname{obj}\left(\mathcal{M} f_{m}\right), \nabla \in \underline{Q}(M)$. Conversely, any $\mathcal{M} f_{m}$-natural operator $D: Q \rightsquigarrow \otimes^{q+1} T^{*} \otimes \otimes^{q} T$ defines an $\mathcal{M} f_{m}$-natural operator $C^{D}: Q \rightsquigarrow\left(\otimes^{k} T^{*}, \otimes^{q+1} T^{*} \otimes \otimes^{q} T\right), C_{M}^{D}(\nabla)(v)=D_{M}(\nabla)(x), v \in$ $\otimes^{k} T_{x}^{*} M, x \in M, M \in \operatorname{obj}\left(\mathcal{M} f_{m}\right), \nabla \in \underline{Q}(M)$. Moreover, the correspondences $C \rightarrow D^{C}$ and $D \rightarrow C^{D}$ are mutually inverse.

Proof. Let $C: Q \rightsquigarrow\left(\otimes^{k} T^{*}, \otimes^{q+1} T^{*} \otimes \otimes^{q} T\right)$ be an $\mathcal{M} f_{m}$-natural operator. We use the notation from the proof of Lemma 1. The invariance of $C$ with respect to the homotheties $\frac{1}{t} i d_{\mathbb{R}^{m}}$ gives the homogeneity condition

$$
t C_{\mathbb{R}^{m}}\left(\left(\nabla_{j k ; \alpha}^{i}\right)\right)(v)=C_{\mathbb{R}^{m}}\left(\left(t^{1+|\alpha|} \nabla_{j k ; \alpha}^{i}\right)\right)\left(t^{k} v\right) .
$$

Then, (if $k \geq 2) C_{\mathbb{R}^{m}}\left(\left(\nabla_{j k ; \alpha}^{i}\right)\right)(v)=C_{\mathbb{R}^{m}}\left(\left(\nabla_{j k ; \alpha}^{i}\right)\right)(0)$ because of the homogeneous function theorem. So, $C_{M}(\nabla)(v)=C_{M}(\nabla)(0)$ for all $M \in \operatorname{obj}\left(\mathcal{M} f_{m}\right), \nabla \in \underline{Q}(M)$, $v \in \otimes^{k} T_{x}^{*} M, x \in M$. Consequently, the correspondences $C \rightarrow D^{C}$ and $D \rightarrow C^{D}$ are mutually inverse.

Lemma 3. If $k \geq 3$, the $\mathcal{M} f_{m}$-natural operators $C: Q \rightsquigarrow\left(\otimes^{k} T^{*}, \otimes^{k+2} T^{*}\right)$ are in bijection with the pairs $(E, F)$ of $\mathcal{M} f_{m}$-natural operators $E: Q \rightsquigarrow \otimes^{k+2} T^{*}$ and $F: Q \rightsquigarrow \otimes^{k+2} T^{*} \otimes \otimes^{k} T$.

More precisely, any $\mathcal{M} f_{m}$-natural operator $C: Q \rightsquigarrow\left(\otimes^{k} T^{*}, \otimes^{k+2} T^{*}\right)$ defines an $\mathcal{M} f_{m}$-natural operator $E^{C}: Q \rightsquigarrow \otimes^{k+2} T^{*}, E_{M}^{C}(\nabla)(x):=C_{M}(\nabla)(0), M \in$ $\operatorname{obj}\left(\mathcal{M} f_{m}\right), x \in M, \nabla \in \underline{Q}(M), 0 \in \otimes^{k} T_{x}^{*} M$ (the zero) and an $\mathcal{M} f_{m}$-natural operator $F^{C}: Q \rightsquigarrow \otimes^{k+2} T^{*} \otimes \otimes^{k} T$,

$$
\left\langle F_{M}^{C}(\nabla)(x), v\right\rangle:=\left.\frac{d}{d \tau}\right|_{\tau=0} C_{M}(\nabla)(\tau v),
$$

$M \in \operatorname{obj}\left(\mathcal{M} f_{m}\right), \nabla \in \underline{Q}(M), v \in \otimes^{k} T_{x}^{*} M, x \in M, 0 \in \otimes^{k} T_{x}^{*} M$ (the zero). Conversely, any pair $(E, F)$ of an $\mathcal{M} f_{m}$-natural operator $E: Q \rightsquigarrow \otimes^{k+2} T^{*}$ and 
an $\mathcal{M} f_{m}$-natural operator $F: Q \rightsquigarrow \otimes^{k+2} T^{*} \otimes \otimes^{k} T$ defines an $\mathcal{M} f_{m}$-natural operator $C^{(E, F)}: Q \rightsquigarrow\left(\otimes^{k} T^{*}, \otimes^{k+2} T^{*}\right)$,

$$
C_{M}^{(E, F)}(\nabla)(v):=E_{M}(\nabla)(x)+\left\langle F_{M}(\nabla)(x), v\right\rangle,
$$

$M \in \operatorname{obj}\left(\mathcal{M} f_{m}\right), \nabla \in \underline{Q}(M), v \in \otimes^{k} T_{x}^{*} M, x \in M$. Moreover, if $k \geq 3$, the correspondences $C \rightarrow\left(\bar{E}^{C}, F^{C}\right)$ and $(E, F) \rightarrow C^{(E, F)}$ are mutually inverse.

If $k=2$, the $\mathcal{M} f_{m}$-natural operators $C: Q \rightsquigarrow\left(\otimes^{2} T^{*}, \otimes^{4} T^{*}\right)$ are in bijection with the triples $(E, F, G)$ of $\mathcal{M} f_{m}$-natural operators $E: Q \rightsquigarrow \otimes^{4} T^{*}$ and $F: Q \rightsquigarrow$ $\otimes^{4} T^{*} \otimes \otimes^{2} T$ and $\mathcal{M} f_{m}$-natural transformations $G: \otimes^{2} T^{*} \rightarrow \otimes^{4} T^{*}$.

More precisely, any $\mathcal{M} f_{m}$-natural operator $C: Q \rightsquigarrow\left(\otimes^{2} T^{*}, \otimes^{4} T^{*}\right)$ defines an $\mathcal{M} f_{m}$-natural operator $E^{C}: Q \rightsquigarrow \otimes^{4} T^{*}$ and an $\mathcal{M} f_{m}$-natural operator $F^{C}$ : $Q \rightsquigarrow \otimes^{4} T^{*} \otimes \otimes^{2} T$ (the same as above but for $k=2$ ) and an $\mathcal{M} f_{m}$-natural transformation $G^{C}: \otimes^{2} T^{*} \rightarrow \otimes^{4} T^{*}$,

$$
G_{M}^{C}(v):=\left.\frac{1}{2} \frac{d^{2}}{d \tau^{2}}\right|_{\tau=0} C_{M}(\nabla)(\tau v)
$$

$M \in \operatorname{obj}\left(\mathcal{M} f_{m}\right), v \in \otimes^{2} T_{x}^{*} M, x \in M, \nabla \in \underline{Q}(M)$ (the definition of $G_{M}^{C}(v)$ is independent of the choice of $\nabla \in Q(M))$. Conversely, any triple $(E, F, G)$ of an $\mathcal{M} f_{m}$-natural operator $E: Q \rightsquigarrow \bar{\otimes}^{4} T^{*}$ and an $\mathcal{M} f_{m}$-natural operator $F: Q \rightsquigarrow$ $\otimes^{4} T^{*} \otimes \otimes^{2} T$ and an $\mathcal{M} f_{m}$-natural transformation $G: \otimes^{2} T^{*} \rightarrow \otimes^{4} T^{*}$ defines an $\mathcal{M} f_{m}$-natural operator $C^{(E, F, G)}: Q \rightsquigarrow\left(\otimes^{2} T^{*}, \otimes^{4} T^{*}\right)$,

$$
C_{M}^{(E, F, G)}(\nabla)(v):=E_{M}(\nabla)(x)+\left\langle F_{M}(\nabla)(x), v\right\rangle+G_{M}(v),
$$

$M \in \operatorname{obj}\left(\mathcal{M} f_{m}\right), \nabla \in Q(M), v \in \otimes^{2} T_{x}^{*} M, x \in M$. Moreover, if $k=2$, the correspondences $\left.C \rightarrow{ }^{(E}{ }^{C}, F^{C}, G^{C}\right)$ and $(E, F, G) \rightarrow C^{(E, F, G)}$ are mutually inverse.

Proof. Let $C: Q \rightsquigarrow\left(\otimes^{k} T^{*}, \otimes^{k+2} T^{*}\right)$ be an $\mathcal{M} f_{m}$-natural operator. We use the notations from the proof of Lemma 1. The invariance of $C$ with respect to the homotheties gives the homogeneity condition

$$
t^{k+2} C_{\mathbb{R}^{m}}\left(\left(\nabla_{j k ; \alpha}^{i}\right)\right)(v)=C_{\mathbb{R}^{m}}\left(\left(t^{1+|\alpha|} \nabla_{j k ; \alpha}^{i}\right)\right)\left(t^{k} v\right) .
$$

Then, by virtue of the homogeneous function theorem and the Taylor formula for $\tau \rightarrow C_{\mathbb{R}^{m}}\left(\left(\nabla_{j k ; \alpha}^{i}\right)\right)(\tau v)$ in $\tau_{0}=0$ and evaluated at $\tau=1$, we have

$$
\begin{gathered}
C_{\mathbb{R}^{m}}\left(\left(\nabla_{j k ; \alpha}^{i}\right)\right)(v)=C_{\mathbb{R}^{m}}\left(\left(\nabla_{j k ; \alpha}^{i}\right)\right)(0)+\left.\frac{d}{d \tau}\right|_{\tau=0} C_{\mathbb{R}^{m}}\left(\left(\nabla_{j k ; \alpha}^{i}\right)\right)(\tau v) \\
+\left.\frac{1}{2} \frac{d^{2}}{d \tau^{2}}\right|_{\tau=0} C_{\mathbb{R}^{m}}\left(\left(\nabla_{j k ; \alpha}^{i}\right)\right)(\tau v)
\end{gathered}
$$

for any $\left(\nabla_{j k ; \alpha}^{i}\right)$ and $v \in \otimes^{k} T_{0}^{*} \mathbb{R}^{m}$. Moreover, $\left.\frac{d^{2}}{d \tau^{2}}\right|_{\tau=0} C_{\mathbb{R}^{m}}\left(\left(\nabla_{j k ; \alpha}^{i}\right)\right)(\tau v)$ is zero if $k \geq 3$ and it is independent of $\left(\nabla_{j k ; \alpha}^{i}\right)$ if $k=2$. 
So, we have

$$
C_{M}(\nabla)(v)=C_{M}(\nabla)(0)+\left.\frac{d}{d \tau}\right|_{\tau=0} C_{M}(\nabla)(\tau v)+\left.\frac{1}{2} \frac{d^{2}}{d \tau^{2}}\right|_{\tau=0} C_{M}(\nabla)(\tau v)
$$

for any $M \in \operatorname{obj}\left(\mathcal{M} f_{m}\right), \nabla \in \underline{Q}(M), v \in \otimes^{k} T_{x}^{*} M, x \in M$. Moreover, the expression $\left.\frac{d^{2}}{d \tau^{2}}\right|_{\tau=0} C_{M}(\nabla)(\tau v)$ is zero if $k \geq 3$ and it is independent of $\nabla$ if $k=2$. Therefore, the definition of $G_{M}^{C}(v)$ (for $k=2$ ) is really independent of the choice of $\nabla$ and the correspondences $C \rightarrow\left(E^{C}, F^{C}\right)$ and $(E, F) \rightarrow C^{(E, F)}$ for $k \geq 3$ are mutually inverse and the correspondences $C \rightarrow\left(E^{C}, F^{C}, G^{C}\right)$ and $(E, F, G) \rightarrow$ $C^{(E, F, G)}$ for $k=2$ are also mutually inverse.

\section{THE MAIN RESULT}

The main result of the present note is the following theorem, which is an immediate consequence of Proposition 1 and Lemmas 1, 2, and 3.

Theorem 1. (1) If $k \geq 3$, the $\mathcal{M} f_{m}$-natural operators $B: Q \rightsquigarrow Q\left(\otimes^{k} T^{*}\right)$ are in bijection with the systems $D=\left(D_{j}\right)_{j=1, \ldots, 5}$ of $\mathcal{M} f_{m}$-natural operators $D_{1}: Q \rightsquigarrow \otimes^{2} T^{*} \otimes T, D_{2}: Q \rightsquigarrow \otimes^{k+2} T^{*}, D_{3}: Q \rightsquigarrow \otimes^{k+2} T^{*} \otimes$ $\otimes^{k} T, D_{4}: Q \rightsquigarrow \otimes^{k+1} T^{*} \otimes \otimes^{k} T$ and $D_{5}: Q \rightsquigarrow \otimes^{k+1} T^{*} \otimes \otimes^{k} T$.

(2) If $k=2$, the $\mathcal{M} f_{m}$-natural operators $B: Q \rightsquigarrow Q\left(\otimes^{k} T^{*}\right)$ are in bijection with the systems $D=\left(D_{j}\right)_{j=1, \ldots, 6}$ of $\mathcal{M} f_{m}$-natural operators $D_{1}: Q \rightsquigarrow$ $\otimes^{2} T^{*} \otimes T, D_{2}: Q \rightsquigarrow \otimes^{4} T^{*}, D_{3}: Q \rightsquigarrow \otimes^{4} T^{*} \otimes \otimes^{2} T, D_{4}: Q \rightsquigarrow$ $\otimes^{3} T^{*} \otimes \otimes^{2} T, D_{5}: Q \rightsquigarrow \otimes^{3} T^{*} \otimes \otimes^{2} T$ and $\mathcal{M} f_{m}$-natural transformations $D_{6}: \otimes^{2} T^{*} \rightarrow \otimes^{4} T^{*}$.

\section{REFERENCES}

[1] J. Gancarzewicz and I. Koláŕ, "Some gauge-natural operators on linear connections," Monatsh. Math., vol. 111, pp. 23-33, 1991.

[2] I. Kolář, P. W. Michor, and J. Slovák, Natural Operations in Differential Geometry. Berlin: Springer-Verlag, 1993.

[3] M. Kureš, "Natural lifts of classical linear connections to the cotangent bundle," in Suppl. Rend. Mat. Palermo II, vol. 43, 1996, pp. 181-187.

Authors' addresses

Jan Kurek

Institute of Mathematics, Maria Curie-Sklodowska University, pl. Marii Curie-Sklodowskiej 1, Lublin, Poland

E-mail address: kurekehektor.umcs. lublin.pl

\section{Włodzimierz M. Mikulski}

Institute of Mathematics, Faculty of Mathematics and Computer Science, Jagiellonian University, ul. S. Łojasiewicza 6, Kraków, Poland

E-mail address: Wlodzimierz.Mikulski@im.uj.edu.pl 